\title{
Flexible rods and the case for dynamic stabilization
}

\author{
Jason M. Highsmith, M.D., Luis M. Tumialán, M.D., and Gerald E. Rodts JR., M.D. \\ Department of Neurosurgery, Emory University School of Medicine, Atlanta, Georgia
}

\begin{abstract}
$\checkmark$ The widespread use of instrumentation in the lumbar spine has led to high rates of fusion. This has been accompanied by a marked rise in adjacent-segment disease, which is considered to be an increasingly common and significant consequence of lumbar or lumbosacral fusion. Numerous biomechanical studies have demonstrated that segments fused with rigid metallic fixation lead to significant amounts of supraphysiological stress on adjacent discs and facets. The resultant disc degeneration and/or stenosis may require further surgical intervention and extension of the fusion to address symptomatic adjacent-segment disease.

Recently, dynamic stabilization implants and disc arthroplasty have been introduced as an alternative to rigid fixation. The scope of spinal disease that can be treated with this novel technology, however, remains limited, and these treatments may not apply to patients who still require rigid stabilization and arthrodesis.

In the spectrum between rigid metallic fixation and motion-preserving arthroplasty is a semirigid type of stabilization in which a construct is used that more closely mirrors the modulus of elasticity of natural bone. After either interbody or posterolateral arthrodesis is achieved, the fused segments will not generate the same adjacent-level forces believed to be the cause of adjacent-segment disease. Although this form of arthrodesis does not completely prevent adjacent-segment disease, the dynamic component of this stabilization technique may minimize its occurrence.

The authors report their initial experience with the use of posterior dynamic stabilization in which polyetheretherketone rods were used for a posterior construct. The biomechanics of dynamic stabilization are discussed, clinical indications are reviewed, and case studies for its application are presented.
\end{abstract}

KEY WORDS • adjacent-segment disease • dynamic stabilization • polyetheretherketone

$\mathrm{T}$ HE use of metallic instrumentation for scoliotic spinal deformity and trauma has been well established for decades. The widespread use of rigid instrumentation in the treatment of degenerative lumbar disorders, however, is a relatively newer concept.

Historically, rates for uninstrumented lumbar posterolateral fusion or even interbody fusion have been less than satisfactory. With the introduction in the US of rigid pedicle screw/rod fixation in the lumbar spine in the 1990s, arthrodesis rates in the treatment of degenerative disc disease and spondylolisthesis have improved dramatically. 7,8,10,27,30,41 In addition to higher rates of arthrodesis, rigid instrumentation has enabled surgeons to maintain, improve, or fully reduce spondylolisthesis outright, and these devices have allowed for very aggressive strategies for decompression. The attitudes of surgeons have changed as well; many no longer use rigid external immobilization devices postoperatively, or they use them for a shorter duration. Potential adverse effects of rigid instrumentation and fusion in the lumbar spine, however, are beginning to be recognized.

Concomitant with the meteoric rise in the use of rigid pedicle screw instrumentation has been an increased prevalence of disc degeneration, new spondylolisthesis, disc herniation, or spinal canal stenosis at levels adjacent to the fused segments. The incidence of adjacent-level disease in

Abbreviations used in this paper: $\mathrm{CT}=$ computed tomography; PEEK = polyetheretherketone. patients who have undergone instrumented fusion has been reported to be as high as 35 to $45 \%$. $^{25,35}$ The rate at which this disorder can develop is alarming, especially when compared with the slow progression in patients following an "old-fashioned" onlaid posterolateral fusion without instrumentation. Many surgeons suspect that the degree of stiffness of the instrumented levels relates directly to increased stress on adjacent discs and facet joints. ${ }^{2,5,15}$ These increased loads over time lead to segment hypermobility, facet hypertrophy, osteophyte formation, and stenosis. ${ }^{5}$ Other factors contributing to adjacent-level disease that must be considered include cephalad facet injury and/or partial resection during the placement of the cephalad-most screws, muscle denervation, and the natural history of the underlying disease process. Many surgeons have witnessed a domino-like occurrence of adjacent-level disease, with some patients requiring extension of a fusion construct at serial intervals.

The degree of stiffness of a pedicle screw/rod construct is extremely supraphysiological compared with the modulus of elasticity of an uninstrumented mature posterolateral or Hibbs (interlaminar) arthrodesis, let alone native bone. Nonmalleable constructs of stainless steel or titanium are probably far more rigid than needed to augment fusion. There is presumably an optimum degree of stiffness that would promote fusion while lowering the rate and incidence of adjacent-level disease.

A new generation of spinal implants made of the semicrystalline thermoplastic polymer PEEK has been devel- 

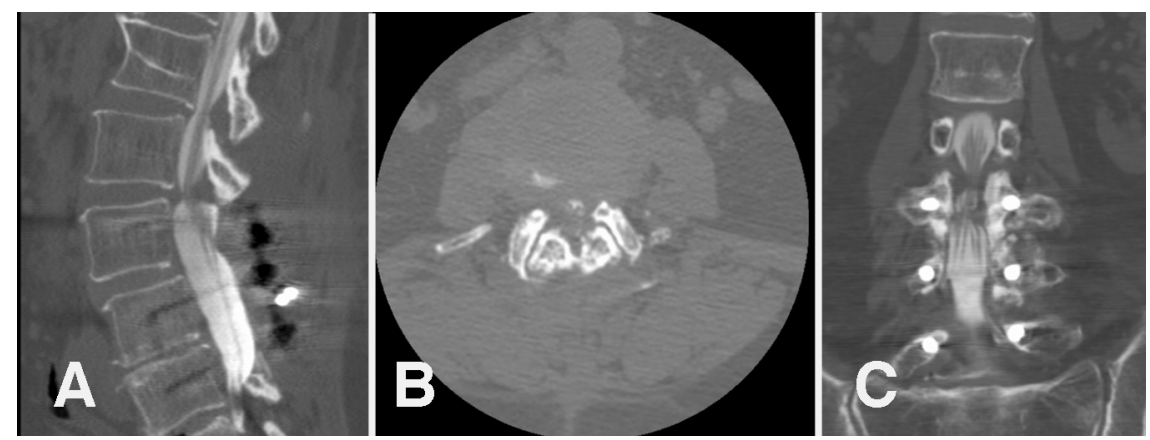

FIG. 1. Case 1. Sagittal, axial, and coronal CT myelograms demonstrating severe adjacent-level stenosis at L2-3 above an L3-5 pedicle screw fusion in which a traditional titanium rod was used.

oped. A well-known substrate in spinal surgery, PEEK has been used extensively in cervical and lumbar interbody constructs. This polymer has a modulus of elasticity between that of cortical and cancellous bone, thus mimicking the load characteristics of the native environment.

Recently, rods made of PEEK have been introduced as a semirigid alternative to their nonmalleable stainless steel or titanium counterparts. The PEEK rod attaches to a modified top-loading multiaxial metallic screw (CD Horizon Legacy; Medtronic Sofamor Danek). These rods allow some motion, but resist marked flexion, extension, axial loading, and lateral rotation. Laboratory testing has demonstrated their ability to reduce stress and hypermobility at adjacent levels compared with titanium screw/rod constructs. Furthermore, PEEK is a radiolucent material that will not interfere with the plain x-ray films or CT scans that are needed for evaluation of fusion.

We suggest that the semirigid hybrid of titanium pedicle screws and PEEK rods may have several clinical indications. The first scenario is the de novo treatment of spinal instability (spondylolisthesis, recurrent disc herniation, or degenerative disc disease), in which a solid osseous arthrodesis is desired, with less potential stress on adjacent lumbar spinal segments.

A second clinical indication is for patients who have already undergone an instrumented fusion but in whom adjacent-level disease (instability, disc deterioration, stenosis, disc herniation, and so on) has developed. In this scenario, stabilization adjacent to the previous fusion is desired, but a more dynamic strategy may prevent a subsequent operation at neighboring levels. There are several options at this stage. One could consider complete explantation of indwelling titanium rods and screws, with placement of new titanium screws and PEEK rods only at the new level being treated. One could also consider leaving in place the existing titanium instrumentation but "piggybacking" onto the cephalad aspect of the construct with a PEEK rod and new titanium screws at the adjacent level. Still further, one could consider leaving in place all of the existing screws, removing the titanium rods, placing the new levels of screws, and then connecting the entire construct with PEEK rods.

A third potential use of a hybrid PEEK rod/titanium screw construct is to create a tension band. This can be used to stabilize the spine in a patient with mobile or fixed spondylolisthesis and stenosis. In this clinical scenario, an osseous arthrodesis may not be necessary following a fa- cet-sparing bilateral laminectomy. The creation of a posterior tension band may limit progression of the spondylolisthesis and reduce the incidence of radicular symptoms or back pain. An elderly patient with a low-grade spondylolisthesis, stenosis, and minimal movement on preoperative lateral flexion-extension x-ray films would be a potential candidate.

In this paper, we present three types of patients in whom semirigid fixation may prove to have an advantage over the traditional titanium screw/rod construct.

\section{Illustrative Cases}

\section{Case 1}

This 65-year-old woman who had undergone instrumented L3-5 fusion 8 months previously presented with new-onset neurogenic claudication. A CT myelogram demonstrated severe stenosis at L2-3 caused by diffuse disc bulge, severe ligamentum flavum hypertrophy, and facet overgrowth (Fig. 1). With nonsurgical management having failed, the patient underwent explantation of her hardware and fusion of the adjacent level with PEEK rods placed from L-2 to L-3. She also received autologous bone and tricalcium phosphate hydroxyapatite (Mastergraft; Sofamor Danek) wrapped in a collagen sponge along with recombinant human bone morphogenetic protein-2. The patient experienced immediate relief of her stenotic symptoms and is showing signs of bone fusion growth.

\section{Case 2}

This 48-year-old man presented with severe degenerative disc disease at L5-S1, with marked stenosis at L4-5. Given the patient's young age and active lifestyle, the decision was made to use a hybrid construct to perform rigid fixation at the caudal level and dynamic stabilization at the adjacent level. The patient underwent transforaminal lumbar interbody fusion at L5-S1 and pedicle screw placement from L-4 to S-1, with PEEK rod placement for stabilization (Fig. 2).

\section{Case 3}

This 80-year-old woman presented with neurogenic claudication but minimal back pain. Her imaging studies revealed severe L4-5 stenosis and a Grade I spondylolisthesis. She had a tall disc space and demonstrated slight 


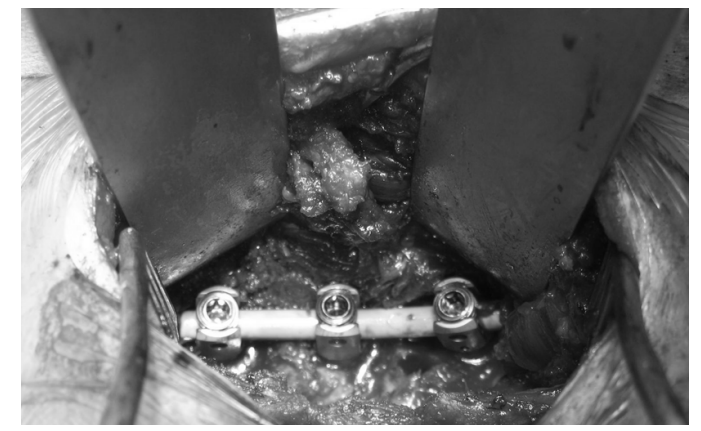

FIG. 2. Case 2. Intraoperative photograph showing hardware implanted on the left side at L4-S1.

movement of the segments on flexion-extension plain $\mathrm{x}$ ray films. Although a fusion was not indicated, there was obvious concern over instability following a simple laminectomy. She underwent laminectomy for decompression and placement of a pedicle screw/PEEK rod construct for stabilization with no arthrodesis. Postoperative radiographs showed preserved alignment (Fig. 3).

\section{Discussion}

Disc arthroplasty has been offered as an alternative to fusion in hopes of preserving motion, alleviating pain, and preventing rapid development of adjacent-level disease. The latter effect, however, has not yet been clearly demonstrated, nor has an overall advantage been found for lumbar disc arthroplasty over current interbody fusion techniques. Furthermore, disc arthroplasty in the lumbar spine has a very narrow set of clinical and radiographic indications. For example, it cannot be used to treat common diseases such as stenosis or spondylolisthesis. Anatomically, lumbar disc arthroplasty is limited to anterior disease of the lower lumbar spine, and even then is only approved by the Food and Drug Administration for a single level.

Dynamic posterior lumbar instrumentation has the potential to overcome many of these limitations. Lumbar dynamic stabilization has been used extensively outside of North America for more than a decade. ${ }^{33}$ The Graf posterior ligamentoplasty system ${ }^{18}$ was developed to reduce rotational movement in the spine by locking the facets in extension, but allowed compression of the posterior anulus. The latter effect has been linked to painful load bearing. ${ }^{33}$

The Dynesys system (Zimmer Spine) is a posterior pedicle screw and cord system designed to create a tension band that constrains motion. In this system, a flexion-limiting fabric cord is surrounded by an extension-limiting polymer tube wedged between adjacent-level pedicle screws. The device has been shown to reduce pressure in the disc by limiting flexion $30 \% .{ }^{36}$ However, it has shown implant failure rates as high as $17 \%{ }^{37}$ and reoperation rates of $19 \%$ during a mean 2-year follow-up duration. ${ }^{19}$ Range of motion in the Dynesys construct depends on the length of the polymer implant, which is cut to the desired length at the time of implantation. ${ }^{32}$ Making the implant too long may cause a focal kyphosis, to which poor outcomes have been attributed. ${ }^{39}$

The primary goal of spinal instrumentation in fusion is to immobilize two or more segments to create an environ-

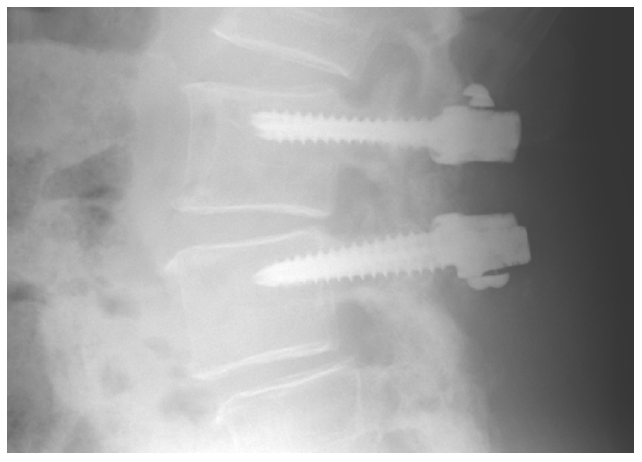

FIG. 3. Case 3. Lateral radiograph showing titanium pedicle screw instrumentation joined by PEEK rods. Note the radiolucent caps on the ends of the rods.

ment conducive to healing of the interbody or posterolateral bone graft. The years of refinement of these techniques and the addition of biological factors such as recombinant human bone morphogenic protein have continued to increase fusion rates. ${ }^{9}$ The consequence of this success has been the more common and more rapid development of adjacent-segment disease. . $^{115-17,20,22-24,34,38}$

The supraphysiological biomechanical stresses created by rigid metallic fixation of the lumbar spine appear to be the central cause of adjacent-segment disease.,14-17 Although there is little question that pedicle screw/rod constructs optimize fusion, such a rigid system may become more of a liability than an asset in the long term after a bone fusion is achieved. Although the exact origin of adjacent-segment disease remains uncertain, biomechanical studies have established that fusion of adjacent segments shifts the center of rotation posteriorly, which in turn increases the stress on facets and discs at adjacent, unfused segments. ${ }^{24}$ Such a shift in the center of rotation may be necessary for fusion to occur, but the extent of rigidity needed remains in question. Furthermore, the onset of symptomatic adjacent-segment disease may be considerably delayed from the time of initial surgery. ${ }^{1,17}$ In a series of 18 patients, Lee ${ }^{24}$ reports the onset of symptomatic adjacent-segment disease after a mean of 8.5 symptom-free years (range 1-38 years). Thus, the fundamental question becomes this: what degree of rigidity is needed in lumbar fixation to establish a successful bone fusion, and for how long?

In considering this question, two viable options to minimize the risk of adjacent-segment disease become clear. The first option is the continued use of current rigid fixation systems, followed by explantation after a radiographically confirmed fusion is achieved. In this scenario, instead of a titanium construct, the posterolateral or interbody fusion mass would now exert the forces on adjacent levels. The main disadvantage of this approach is the need for a second operation.

The second option is the use of a less rigid construct that would allow for adequate immobilization of adjacent segments for fusion but would not exert the supraphysiological stresses on adjacent facets and intervertebral discs associated with titanium rods. Although other semirigid systems have been previously reported, ${ }^{21,28}$ the ideal construct would mirror the modulus of elasticity of bone, suf- 
ficiently limiting motion of the segments for fusion. The less rigid construct would prevent the stress shielding that occurs with titanium constructs and would allow the fusion mass to exert stresses that are more physiological to adjacent levels.

Polyetheretherketone is a nonresorbable, semicrystalline, polyaromatic linear polymer, which has been used extensively as a spacer for interbody fusion in both the cervical and lumbar spine. ${ }^{6,11-13,29}$ The biomechanics of changing this polymer from a spacer into a rod, specifically the flexural modulus and fatigue strength, has wellestablished precedents in the orthopedic literature, where PEEK polymers have been used as femoral stems in total hip arthroplasty. ${ }^{3,4,26,31}$ Because PEEK's modulus of elasticity is similar to that of bone (approximately $17 \mathrm{GPa}$ ) ${ }_{40}$ the use of this polymer as part of a pedicle screw/rod construct would offer adequate rigidity for fusion to occur but would not exert the stresses created by a titanium construct. The putative benefit of such a polymer rod in the lumbar spine is immobilization for fusion in the short term, while minimizing the risk of adjacent-segment disease in the long term.

The PEEK rods can reduce stress on the screw-bone anchor points and potentially reduce pedicle fractures and construct failure. The compliance of a PEEK rod allows some loading of the interbody construct and can theoretically increase fusion rates by allowing more contact between the endplate and graft. Finally, the implant is associated with reduced scatter and artifact on all modalities of spine imaging.

Although very much in its infancy, the use of PEEK rods for a posterior construct in lumbar fusion represents one of the many viable modalities available to surgeons to begin to address the causative factors of adjacent-segment disease. For this proposed method to be adopted, practitioners will first need to establish fusion rates that are equivalent to existing technologies and then demonstrate a decrease in the incidence of adjacent-segment disease.

\section{Conclusions}

The PEEK rods described here bridge the gap between fully dynamic constructs such as disc arthroplasty, constrained dynamic constructs such as Dynesys, and rigid fixation systems such as titanium pedicle screw/rod constructs. The use of PEEK rods for fusion in the lumbar spine addresses the causative factors of adjacent-segment disease, but this theoretical benefit remains to be proven. Further studies are required to establish fusion rates with this technology and to demonstrate a decreased incidence of adjacent-segment disease with this form of semirigid stabilization.

\section{Disclosure}

Dr. Rodts acts as a consultant for Medtronic Sofamor Danek, which provided no financial or material support for this paper.

\section{References}

1. Aiki H, Ohwada O, Kobayashi H, Hayakawa M, Kawaguchi S, Takebayashi T, et al: Adjacent segment stenosis after lumbar fusion requiring second operation. J Orthop Sci 10:490-495, 2005
2. Akamaru T, Kawahara N, Tim Yoon S, Minamide A, Su Kim $\mathrm{K}$, Tomita K, et al: Adjacent segment motion after a simulated lumbar fusion in different sagittal alignments: a biomechanical analysis. Spine 28:1560-1566, 2003

3. Akay M, Aslan N: An estimation of fatigue life for a carbon fiber/poly ether ether ketone hip joint prosthesis. Proc Inst Mech Eng [H] 209:93-103, 1995

4. Albert K, Schledjewski R, Harbaugh M, Bleser S, Jamison R, Friedrich K: Characterization of wear in composite material orthopaedic implants. Part II: the implant/bone interface. Biomed Mater Eng 4:199-211, 1994

5. Bastian L, Lange U, Knop C, Tusch G, Blauth M: Evaluation of the mobility of adjacent segments after posterior thoracolumbar fixation: a biomechanical study. Eur Spine J 10:295-300, 2001

6. Boakye M, Mummaneni PV, Garrett M, Rodts G, Haid R: Anterior cervical discectomy and fusion involving a polyetheretherketone spacer and bone morphogenetic protein. J Neurosurg Spine 2:521-525, 2005

7. Bono CM, Lee CK: Critical analysis of trends in fusion for degenerative disc disease over the past 20 years: influence of technique on fusion rate and clinical outcome. Spine 29: 455-463, 2004

8. Boos N, Webb JK: Pedicle screw fixation in spinal disorders: a European view. Eur Spine J 6:2-18, 1997

9. Brantigan JW, Neidre A, Toohey JS: The Lumbar I/F Cage for posterior lumbar interbody fusion with the variable screw placement system: 10-year results of a Food and Drug Administration clinical trial. Spine J 4:681-688, 2004

10. Bridwell KH, Sedgewick TA, O’Brien MF, Lenke LG, Baldus $\mathrm{C}$ : The role of fusion and instrumentation in the treatment of degenerative spondylolisthesis with spinal stenosis. J Spinal Disord 6:461-472, 1993

11. Cho DY, Lee WY, Sheu PC: Treatment of multilevel cervical fusion with cages. Surg Neurol 62:378-386, 2004

12. Cho DY, Lee WY, Sheu PC, Chen CC: Cage containing a biphasic calcium phosphate ceramic (Triosite) for the treatment of cervical spondylosis. Surg Neurol 63:497-504, 2005

13. Cho DY, Liau WR, Lee WY, Liu JT, Chiu CL, Sheu PC: Preliminary experience using a polyetheretherketone (PEEK) cage in the treatment of cervical disc disease. Neurosurgery 51: 1343-1350, 2002

14. Chosa E, Goto K, Totoribe K, Tajima N: Analysis of the effect of lumbar spine fusion on the superior adjacent intervertebral disk in the presence of disk degeneration, using the three-dimensional finite element method. J Spinal Disord Tech 17: 134-139, 2004

15. Eck JC, Humphreys SC, Hodges SD: Adjacent-segment degeneration after lumbar fusion: a review of clinical, biomechanical, and radiologic studies. Am J Orthop 28:336-340, 1999

16. Etebar S, Cahill DW: Risk factors for adjacent-segment failure following lumbar fixation with rigid instrumentation for degenerative instability. J Neurosurg 90 (2 Suppl):163-169, 1999

17. Ghiselli G, Wang JC, Bhatia NN, Hsu WK, Dawson EG: Adjacent segment degeneration in the lumbar spine. J Bone Joint Surg Am 86:1497-1503, 2004

18. Graf H: [Lumbar instability. Surgical treatment without fusion.] Rachis 412:123-137, 1992 (Fr)

19. Grob D, Benini A, Junge A, Mannion AF: Clinical experience with the Dynesys semirigid fixation system for the lumbar spine: surgical and patient-oriented outcome in 50 cases after an average of 2 years. Spine 30:324-331, 2005

20. Hilibrand AS, Robbins M: Adjacent segment degeneration and adjacent segment disease: the consequences of spinal fusion? Spine J 4 (6 Suppl):190S-194S, 2004

21. Korovessis P, Papazisis Z, Koureas G, Lambiris E: Rigid, semirigid versus dynamic instrumentation for degenerative lumbar spinal stenosis: a correlative radiological and clinical analysis of short-term results. Spine 29:735-742, 2004 
22. Lai PL, Chen LH, Niu CC, Chen WJ: Effect of postoperative lumbar sagittal alignment on the development of adjacent instability. J Spinal Disord Tech 17:353-357, 2004

23. Lai PL, Chen LH, Niu CC, Fu TS, Chen WJ: Relation between laminectomy and development of adjacent segment instability after lumbar fusion with pedicle fixation. Spine 29:2527-2532, 2004

24. Lee CK: Accelerated degeneration of the segment adjacent to a lumbar fusion. Spine 13:375-377, 1988

25. Lehmann TR, Spratt KF, Tozzi JE, Weinstein JN, Reinarz SJ, el-Khoury GY, et al: Long-term follow-up of lower lumbar fusion patients. Spine 12:97-104, 1987

26. Maharaj G, Bleser S, Albert K, Lambert R, Jani S, Jamison R: Characterization of wear in composite material orthopaedic implants. Part I: the composite trunnion/ceramic head interface. Biomed Mater Eng 4:193-198, 1994

27. Mardjetko SM, Connolly PJ, Shott S: Degenerative lumbar spondylolisthesis. A meta-analysis of literature 1970-1993. Spine 19 (20 Suppl):2256S-2265S, 1994

28. Markwalder TM, Wenger M: Dynamic stabilization of lumbar motion segments by use of Graf's ligaments: results with an average follow-up of 7.4 years in 39 highly selected, consecutive patients. Acta Neurochir (Wien) 145:209-214, 2003

29. Mastronardi L, Ducati A, Ferrante L: Anterior cervical fusion with polyetheretherketone (PEEK) cages in the treatment of degenerative disc disease. Preliminary observations in 36 consecutive cases with a minimum 12-month follow-up. Acta Neurochir (Wien) 148:307-312, 2006

30. McAfee PC, Weiland DJ, Carlow JJ: Survivorship analysis of pedicle spinal instrumentation. Spine 16 (8 Suppl): S422S427, 1991

31. Morrison C, Macnair R, MacDonald C, Wykman A, Goldie I, Grant $\mathrm{MH}$ : In vitro biocompatibility testing of polymers for orthopaedic implants using cultured fibroblasts and osteoblasts. Biomaterials 16:987-992, 1995

32. Niosi CA, Zhu QA, Wilson DC, Keynan O, Wilson DR, Oxland TR: Biomechanical characterization of the three-dimensional kinematic behavior of the Dynesys dynamic stabilization system: an in vitro study. Eur Spine J 15:913-922, 2006
33. Nockels RP: Dynamic stabilization in the surgical management of painful lumbar spinal disorders. Spine 30 (16 Suppl): S68S72, 2005

34. Park P, Garton HJ, Gala VC, Hoff JT, McGillicuddy JE: Adjacent segment disease after lumbar or lumbosacral fusion: review of the literature. Spine 29:1938-1944, 2004

35. Rahm MD, Hall BB: Adjacent-segment degeneration after lumbar fusion with instrumentation: a retrospective study. J Spinal Disord 9:392-400, 1996

36. Schmoelz W, Huber JF, Nydegger T, Dipl I, Claes L, Wilke HJ: Dynamic stabilization of the lumbar spine and its effects on adjacent segments: an in vitro experiment. J Spinal Disord Tech 16:418-423, 2003

37. Schnake KJ, Schaeren S, Jeanneret B: Dynamic stabilization in addition to decompression for lumbar spinal stenosis with degenerative spondylolisthesis. Spine 31:442-449, 2006

38. Sengupta DK: Point of view: dynamic stabilization in addition to decompression for lumbar spinal stenosis with degenerative spondylolisthesis. Spine 31:450, 2006

39. Stoll TM, Dubois G, Schwarzenbach O: The dynamic neutralization system for the spine: a multi-center study of a novel non-fusion system. Eur Spine J 11 (2 Suppl): S170-S178, 2002

40. Wenz LM, Merritt K, Brown SA, Moet A, Steffee AD: In vitro biocompatibility of polyetheretherketone and polysulfone composites. J Biomed Mater Res 24:207-215, 1990

41. Zdeblick TA: The treatment of degenerative lumbar disorders. A critical review of the literature. Spine 20 (24 Suppl):126S137S, 1995

Manuscript submitted October 16, 2006.

Accepted November 22, 2006.

Address reprint requests to: Gerald E. Rodts Jr, M.D., Department of Neurosurgery, Emory Orthopedic and Spine Center, 59 Executive Park South, Suite 300, Atlanta, Georgia 30329. email: gerald.rodts@emoryhealthcare.org. 